\title{
Multicanonical Simulation of the van Hemmen Spin Glass*
}

\author{
M. Katoot ${ }^{1}$, U. Hansmann ${ }^{2,3}$ and T. Celik ${ }^{3,4}$
}

\begin{abstract}
We studied the performance of the multicanonical algorithm by simulating the van Hemmen spin glass model and reproduced the exact results for this mean field model. Physical quantities such as energy density, specific heat, susceptibility and order parameters are evaluated at all temperatures.
\end{abstract}

One of the recent and promising developments in computational physics is the use of the multicanonical ensemble[1] for numerical simulations. It was originally developed for systems with first order phase transitions to avoid the supercritical slowing down [2, 3, 4]. Another possible target of the method are systems with conflicting constraints. For low temperatures these systems split into many thermodynamic states, separated by high tunneling barriers. Low temperature canonical simulations tend to get trapped in one of those states. Multicanonical simulations overcome these barriers by connecting back to high temperature states. First promising studies exist for spin glasses [5, 6, [], random ising model [8] and proteins [9]. Here, we test the performance of the multicanonical algorithm against exact results for this kind of systems, namely the van Hemmen spin glass model $[10 \|$.

In this paper, due to limited space, we will not review the multicanonical ensemble. Interested readers should refer to the references [1, 2, 5, 11].

The van Hemmen spin glass model[10] is defined by the hamiltonian:

$$
H=-\frac{J_{0}}{N} \sum_{i, j} S(i) S(j)-\sum_{i, j} J_{i j} S(i) S(j)-h \sum_{i} S(i),
$$

describing $\mathrm{N}$ Ising spins interacting with an external magnetic field $h$ and with each other in pairs $(i, j)$. Via $J_{0}$ a direct ferromagnetic coupling is incorporated. The $J_{i j}$ contain the randomness,

$$
J_{i j}=\frac{J}{N}\left[\xi_{i} \eta_{j}+\xi_{j} \eta_{i}\right],
$$

\footnotetext{
${ }^{1}$ Department of Mathematics and Physical Sciences, Embry-Riddle Aeronautical University, Daytona Beach, FL 32114, USA

${ }^{2}$ Department of Physics, The Florida State University, Tallahassee, FL 32306, USA

${ }^{3}$ Supercomputer Computations Research Institute Tallahassee, The Florida State University, Tallahassee, FL 32306, USA

${ }^{4}$ On leave of absence from Department of Physics Engineering, Hacettepe University, Ankara, Turkey

*To appear in the Proceedings of the Sixth Annual Workshop on Recent Developments in Computer Simulation Studies in Condensed Matter Physics, 22-26 Feb. 1993, Athens, Georgia.
} 
where the $\xi_{i}$ and $\eta_{j}$ are independent and evenly distributed random variables with mean zero and variance one. This distribution of $J_{i j}$ 's is shown [12] to model the RKKY [13] interaction in a real metallic spin glass: symmetric and highly peaked at $J_{i j}=0$. The $J_{i j}$ contain $2 N$ independent random variables and describe therefore a random-site problem, not a random-bond problem like the SK model.

The model has three order parameters,

$$
m_{N}=N^{-1} \sum_{i=1}^{N} S(i), \quad q_{1 N}=N^{-1} \sum_{i=1}^{N} \xi_{i} S(i), \quad q_{2 N}=N^{-1} \sum_{i=1}^{N} \eta_{i} S(i) .
$$

Without a ferromagnetic interaction, i.e., for $J_{0}=0$ the magnetization vanishes $m=$ 0 , and the order parameters $q_{1 N}$ and $q_{2 N}$ are combined to give a more relevant order parameter $Q$ :

$$
Q=N^{-1}<\frac{1}{2}\left(q_{1 N}+q_{2 N}\right)>
$$

from which the thermodynamical quantities can be obtained. This model is exactly solvable without replicas and its main features are consistent with that of a spin glass model with randomness and frustration, except for the metastable state structure. The system actually picks out a Mattis state [14] and lacks therefore a great multiplicity of metastable states which is considered integral to a true spin glass. We like to mention that the van Hemmen model is also very closely related to the Hopfield model [15] which is widely used to model neural networks.

We performed multicanonical simulations of the van Hemmen model on cluster of RISC workstations. Independent gaussian distributions for $\xi_{i}$ and $\eta_{i}$ with mean value zero and variance one were created. Simulations with up to $N=1000$ spins were easily carried out. Thermodynamical averages were evaluated over typically two million iterations, following $2 \times 10^{5}$ iterations of thermalization runs. With this extended statistic and the new method we could go far beyond what was done in earlier work[16]. Let us first concentrate on

Figure 1: Spin glass order parameter distribution.

the pure spin glass case and set the ferromagnetic coupling $J_{0}=0$. Fig.1 shows the distribution of the order parameter $Q$ at all temperatures for $N=1000$ spins $. T_{f} / J=1$ 
Figure 2: Specific heat vs. temperature for several lattice sizes.

is the bifurcation point below which temperature the nonzero ordering sets in and reaches its maximum value $1 / \sqrt{\pi}$ at $T=0$. There is no many-valley structure like the one observed in the multicanonical simulation of Edwards-Anderson model [6]. The internal energy assumes the values 0 at $T=T_{f}$ and $-1 / \pi$ at $T=0$. Fig. 2 displays the specific heat vs. temperature for several lattice sizes. The specific heat is linear at low temperatures, peaks at $0.8<T<1.0$ and vanishes for $T_{f}<T$. The linear behavior of the specific heat indicates the existence of many low lying excited states at low temperatures. Both our values for the internal energy and specific heat reproduce the exact values with high precision.

Next we included the ferromagnetic coupling in the model, with zero external field. The spin glass to ferromagnet transition is supposed to take place in the region $J_{0} \sim J$. We observed that the magnetization jumps and the order respectively vanishes for $J_{0}$ approaching $J$. While at $J_{0} / J=0.6$ the distribution of the order parameter was the same as depicted in Fig.1, for $J_{0} / J=0.9$ it assumed the value $Q=0.34$ at about $T / J \sim 2$ and stayed constant all the way down to $T=0$. The system stays in the metastable spin glass phase, and does not spontaneously jump to the ferromagnetic phase. Such behavior was observed elsewhere [17]. In Fig.3 we show the zero field susceptibility $\chi_{0}(T)$ vs. temperature for several values of the ferromagnetic coupling $J_{0}$. For $T<T_{f}$ the susceptibility develops a plateau, a feature indicating an equilibrium phenomena, and also shared by the Sherrington-Kirkpatrick model. The value of the susceptibility at $T=T_{f}$ agrees well with the analytic solution $\chi_{0}\left(T_{f}\right)=1 /\left(J-J_{0}\right)$. For $T_{f}<T$, we observe a pure Curie-Weiss behavior $\chi_{0}(T)=1 /\left(T-T_{f}\right)$. Entering the region of the metastable spin glass phase mentioned above, around $J_{0} / J=0.6$, the plateau starts getting distorted.

The multicanonical simulation of the van Hemmen model is quite feasible; the phases are clearly observed, main features like linear rising specific heat, the plateau in zero-field susceptibility are easily obtained. Resulting numerical values agree remarkably well with the predicted ones. It is important to notice that these thermodynamic quantities were obtained for all temperatures from one simulation. In our simulations it is straightforward to probe the ground states for systems like the present one which includes randomness and frustration and a continuous energy spectrum. The multicanonical algorithm reveals itself to be a reliable instrument for the simulation of systems with conflicting constraints and a useful tool to study NP-complete systems. 
Figure 3: Susceptibility vs. temperature for several values of the ferromagnetic coupling.

\section{Acknowledgments}

This research project was partially funded by the Department of Energy under contract DE-FC05-85ER2500 and the NATO Science Program. T.C. was supported by TUBITAK of Turkey and U.H by the Deutsche Forschungsgemeinschaft under contract H180411-1.

\section{References}

[1] B. Berg and T. Neuhaus, Phys. Lett. B267 (1991) 249.

[2] B. Berg and T. Neuhaus, Phys. Rev. Lett. 68 (1992) 9.

[3] B. Berg, U. Hansmann, and T. Neuhaus, Z. Phys. B90 (1993) 229.

[4] W. Janke, B. Berg and M. Katoot, Nucl. Phys. B382 (1992) 649.

[5] B. Berg and T. Celik, Phys. Rev. Lett. 69 (1992) 2292.

[6] B. Berg and T. Celik, Int. J. Mod. Phys. C 3 (1992) 1251.

[7] B. Berg, T. Celik, U. Hansmann, FSU-SCRI-92-121, to appear in Europhys. Lett.

[8] E. Marinari and G. Parisi, Europhys. Lett. 19 (1992) 451.

[9] U. Hansmann and Y. Okamoto, FSU-SCRI-93-12, submitted to J. Comp. Chem.

[10] J.L. van Hemmen, Phys. Rev. Lett., 49;

A.C.D. van Enter and J.L. van Hemmen, Phys. Rev. A 29 (1984), 355;

J.L. van Hemmen, A.C.D. van Enter and J. Canisius, Z.Phys. B 50 (1983) 311.

[11] B. Berg, U. Hansmann and T. Neuhaus, Phys. Rev. B47 (1993), 497.

[12] K. Binder and K. Schroder, Phys. Rev. B 14 (1976) 2142.

[13] M.A. Ruderman and C. Kittel, Phys. Rev. 96 (1954) 99; T. Kasuya, Prog. Theor. Phys. 16 (1956) 45; K. Yosida, Phys. Rev. 106 (1957) 893.

[14] D.C. Mattis, Phys. Lett. 56 (1976) 421.

[15] J.J. Hopfield, Proc. Natl. Acad. Sci. USA 79 (1982) 2554. 
[16] I. Morgenstern and J.L. van Hemmen, Phys. Rev. B 32 (1985), 6058.

[17] R. B. Griffith, C.-Y. Weng and J. S. Langer, Phys. Rev. 149 (1966) 301. 